\section{OP0176 RNA SEQUENCING DETECTION OF GENE DYSREGULATION IN B CELLS SORTED FROM SALIVARY GLAND TISSUE AND PERIPHERAL BLOOD REVEALS NEW PATHWAYS INVOLVED IN SJÖGREN'S SYNDROME PATHOPHYSIOLOGY}

E. Rivière ${ }^{1}$, N. Tchitchek ${ }^{1}$, G. Nocturne ${ }^{1,2}$, J. Pascaud ${ }^{1}$, S. Boudaoud ${ }^{1}$, A. Thai ${ }^{3}$,

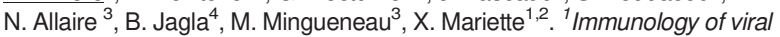
Infections and Autoimmune Diseases, IDMIT, CEA - Université Paris SudINSERM U1184, Le Kremlin Bicêtre and Fontenay aux Roses; ${ }^{2}$ Rheumatology, Université Paris Sud, Le Kremlin Bicêtre, France; ${ }^{3}$ Immunology Research, Biogen, Cambridge, USA; ${ }^{4}$ Biomarker Discovery Platform UtechS CB, Hub de Bioinformatique et biostatistique C3IB, Institut Pasteur, Paris, France

Background: Primary Sjögren's syndrome (pSS) is a chronic auto-immune disorder characterised by lymphocytic infiltrates and destruction of the salivary glands (SG). Chronic B cell activation, the secretion of autoantibodies and the critical role of BAFF have been demonstrated. However, mechanisms leading to B cells dysregulation remain partially understood.

Objectives: To establish transcriptomic maps of the B cells sorted from the SG and from blood using RNASeq analysis.

Methods: Patients had pSS according to 2016 EULAR/ACR criteria and controls had sicca symptoms without any antibodies and with normal SG biopsy. B cells were sorted from SG biopsies and from blood using a FACS ARIA. Total RNASeq profiling was performed using MiSeq (lllumina). Statistical analysis (DESeq2) identified differentially expressed genes between pSS and controls in B cells sorted from SG (9 pSS and 4 controls), from blood (16 pSS and 7 controls); and between $B$ cells sorted from $S G$ and blood in the same patients (4 pSS). Functional enrichment analysis was performed using Ingenuity Pathway Analysis. Results: The pSS vs controls comparison in B cells sorted from SG identified upregulated genes involved in activation of $B$ cells including CD48, CD22 and CD40. TLR10, which is involved in innate immunity was also up-regulated in pSS. The analysis of the non-coding expressed RNAs showed an up-regulation of Mir155 which is essential for $B$ cell differentiation and antibody production (table $1 A$ ). In B cells sorted from blood, TLR7 and the downstream signalling molecule IRF7 were up-regulated in pSS. Additionally, IL-6 which is involved in B cells growth was up-regulated (table 1B). Enrichment analysis highlighted EIF2 signalling pathway, interferon (IFN) signalling pathway and role of JAK in IFN signalling. The paired comparison between B cells from SG and from blood identified up regulated genes including CD138, a plasma cell marker, IL-6, TLR5 and IFN induced genes (table 1C). Mir155HG was also up-regulated.

These results need to be confirmed by RT-qPCR and additional analysis of the non-coding expressed RNA is ongoing.

Table 1 Selection of genes differentially expressed between pSS and controls in $B$ cells sorted from biopsy (1A), blood (1B) and between SG and blood B cells from the same pSS patients (1C)

\begin{tabular}{cccc}
\hline & $\begin{array}{c}\text { Gene } \\
\text { Symbol }\end{array}$ & log2 fold-change & p-value \\
\hline 1A & \multicolumn{3}{c}{ Salivary B cells (pSS vs controls) } \\
\cline { 2 - 4 } & CD48 & 2.59 & 0.009 \\
\cline { 2 - 4 } & CD22 & 2.29 & 0.048 \\
\cline { 2 - 4 } & CD40 & 2.64 & 0.017 \\
\cline { 2 - 4 } & TLR10 & 5.67 & 0.002 \\
\cline { 2 - 4 } & Mir155HG & 4.94 & 0.002 \\
\hline 1B & \multicolumn{3}{c}{ Blood B cells (pSS vs controls) } \\
\cline { 2 - 4 } & TLR7 & 1.40 & 0.008 \\
\cline { 2 - 4 } & IRF7 & 0.76 & 0.041 \\
\cline { 2 - 4 } & IL-6 & 1.54 & 0.006 \\
\hline 1C & \multicolumn{3}{c}{ Salivary vs blood B cells in pSS } \\
\cline { 2 - 4 } & CD138 & 6.92 & $9.63 \mathrm{e}-05$ \\
\cline { 2 - 4 } & IL-6 & 3.05 & 0.004 \\
\cline { 2 - 4 } & TLR5 & 8.86 & 0.011 \\
\cline { 2 - 3 } & Mir155 & 3.10 & 06 \\
\hline
\end{tabular}

Conclusions: This study allowed exploring the mechanisms that support B cell activation in pSS focusing on tissue resident and circulating cells. Our data confirmed the $B$ cell activation and differentiation through several markers including $\mathrm{CD} 40, \mathrm{CD} 22, \mathrm{CD} 48, \mathrm{CD} 138$ and highlighted the role of innate immunity with the TLRs and key pathways including IFN and JAK signalling. Lastly, the role of an epigenetic regulation of $\mathrm{lg}$ secretion is suggested in tissue infiltrating B cells through mir155 expression. Precise understanding of these dysregulation should offer development of new targeted therapeutic perspectives for patients.

Acknowledgements: Arthritis Fondation Courtin for providing a PhD fellowship Disclosure of Interest: None declared
DOI: 10.1136/annrheumdis-2018-eular.3315

\section{OP0177 BCL6 IDENTIFIES ECTOPIC GERMINAL CENTRES IN SALIVARY GLAND BIOPSIES IN PRIMARY SJÖGREN'S} SYNDROME PATIENTS

U. Nakshbandi ${ }^{1}$, E.A. Haacke ${ }^{1,2}$, H. Bootsma ${ }^{1}$, F.K.L. Spijkervet ${ }^{3}$, A. Vissink ${ }^{3}$, B. van der Vegt ${ }^{2}$, F.G.M. Kroese ${ }^{1} .{ }^{1}$ Department of Rheumatology and Clinical Immunology, ${ }^{2}$ Department of Pathology and Medical Biology; ${ }^{3}$ Department of Oral and Maxillofacial Surgery, University of Groningen, University Medical Center Groningen, Groningen, Netherlands

Background: Primary Sjögren's syndrome (pSS) patients who exhibit germinal centres (GCs) within salivary gland parenchyma have higher focus scores and present with more disease activity than GC negative pSS patients. Moreover presence of GCs might be of clinical importance for stratification of treatment. However, there is considerable heterogeneity in reported findings concerning the presence of GCs. This can partially be explained by the difficulty in identifying GCs in diagnostic $\mathrm{H}$ and $\mathrm{E}$ sections and the lack of uniform histopathological criteria.

Objectives: The aim of this study was to assess the most appropriate way to identify unequivocally GCs in parotid and labial gland biopsies of pSS patients.

Methods: As part of routine diagnostic work-up for pSS, both parotid and labial salivary gland biopsies were taken from 100 consecutive patients suspected for pSS. Forty-two patients were classified as having pSS according to the ACREULAR criteria, the remaining 58 patients were classified as non-pSS sicca patients. Diagnostic salivary gland biopsies were formalin fixed, paraffin embedded and serially sectioned at 3-4 $\mu \mathrm{m}$ thickness. Sections were stained with $\mathrm{H}$ and $\mathrm{E}$ as well as immunohistochemically for CD3, CD20, CD21, CD45, Ki67 and Bcl6. Presence of GCs, the number of GCs $/ \mathrm{mm}^{2}$ salivary gland parenchyma and level of lymphoid organisation were determined in all sections.

Results: According to diagnostic $\mathrm{H}$ and $\mathrm{E}$ staining, in $15 \%$ and $2 \%$ of pSS patients GCs were present in parotid and labial salivary glands, respectively. Staining for the proliferation marker Ki67 and the GC-B cell associated transcription factor Bcl6, showed higher percentages: $23 \%$ and $25 \%$. Much higher percentages of follicular dendritic cell (FDCs) networks were revealed by CD21L $(45 \%$ and $55 \%$, for parotid and labial glands, respectively) compared to the number of GCs seen in tissue sections stained for Bcl6. Similarly, the median number of $\mathrm{CD} 21^{+} \mathrm{FDC}$ networks $/ \mathrm{mm}^{2}$ was significantly higher than the number of $\mathrm{GCs} / \mathrm{mm}^{2}$ as revealed by $\mathrm{H}$ and E, Ki67 and Bcl6. Careful evaluation of the consecutive sections stained for CD21L and Bcl6, showed that only roughly half of the FDC networks, also harbour GCs. Finally, not all sections that showed clearly defined GCs by Bcl6 staining, also revealed GCs by Ki67 or $\mathrm{H}$ and $\mathrm{E}$ staining.

Conclusions: Due to the difficulty in $\mathrm{GC}$ recognition, use of diagnostic $\mathrm{H}$ and $\mathrm{E}$ leads to an underestimation and incorrect identification of GCs, while using antiCD21L overestimates GC counts. This suggests that, although CD21 ${ }^{+}$FDC networks play an essential role in GC development and T/B cell compartmentalization, positivity for $\mathrm{CD} 21^{+}$FDC networks does not always imply presence of ectopic GCs. Furthermore, since Ki67 is an excellent marker for solely the dark zone of GCs, small GCs can be overlooked while other proliferative areas might be mistakenly identified as GCs. This study shows that staining for Bcl6 allows easy and unequivocal identification of GCs and should therefore be implemented in the histopathological evaluation of salivary gland biopsies of pSS patients.

Disclosure of Interest: None declared

DOI: 10.1136/annrheumdis-2018-eular.3741

\section{OP0178 ANALYSIS OF B-CELLS SUBSETS IN FIRST DEGREE RELATIVES OF PATIENTS WITH SYSTEMIC LUPUS ERYTHEMATOSUS}

A. Ruiz Roman ${ }^{1}$, A. Muñoz Jimenez ${ }^{1}$, J.M. Lucena ${ }^{2}$, M.A. Montes Cano ${ }^{2}$ B. Sanchez Sanchez ${ }^{2}$, N. Garrido Puñal ${ }^{1}$, E. Blanco Alonso ${ }^{1}$, R. Gill ${ }^{1}$, J. Quijada Carrera ${ }^{1}$, E. Rubio Romero ${ }^{1}$, J. Povedano Gomez ${ }^{1}{ }^{1}$ Reumatologia; ${ }^{2}$ Inmunologia, Hospital Universitario Virgen del Rocio, Sevilla, Spain

Background: Systemic lupus erythematosus (SLE) is an autoimmune, multiorgan disease characterised by periods of activity and remission. In lupus, one of the fields that has most helped his knowledge is the study of lymphocyte subpopulations through flow cytometry. Specifically, in the SLE some alterations have been detected at the level of $B$ lymphocytes as the increase of B-cells subsets such as plasmablasts, plasma cells, transitional cells. There are no studies to date that have analysed the behaviour of $B$ cells subsets in first -degree relatives of patients affected by lupus

Objectives: To analyse if there quantitative difference in the B-cells subsets of the first-degree relatives of SLE with respect to the control population (healthy) and the lupus population 